\title{
Highly Enantioselective Biginelli Reaction of Aliphatic Aldehydes Catalyzed by Chiral Phosphoric Acids
}

Yongbiao Guo*

Zhenhua Gao

Xiangyan Meng

Guilan Huang

Hui Zhong

Huilan Yu

Xiaoqin Ding

Hui Tang

Chuanpin Zou*

Synlett 2017, 27, DOI: 10.1055/s-0036-1588853

In the graphical abstract, the structure of compound $\mathbf{2} \mathbf{f}$ has been corrected. 